\title{
Vantagens e entraves do uso da telemedicina no acompanhamento de pacientes com
}

\section{insuficiência cardíaca}

\author{
Advantages and barriers of using telemedicine in the follow-up of patients with heart faillure \\ Ventajas y barreras del uso de la telemedicina en el seguimiento de pacientes con insuficiencia \\ cardíaca
}

Recebido: 29/06/2021 | Revisado: 05/07/2021 | Aceito: 08/07/2021 | Publicado: 19/07/2021

\author{
Maysa Gabriela Costa Cruz \\ ORCID: https://orcid.org/0000-0002-3950-5845 \\ Centro Universitário Uninovafapi, Brasil \\ E-mail: maysagcostac@gmail.com \\ Lívia Ayres de Miranda Cavalcanti \\ ORCID: https://orcid.org/0000-0002-3271-5030 \\ Centro Universitário Uninovafapi, Brasil \\ E-mail: liviaayrescmed@gmail.com \\ Andressa Carneiro D'Albuquerque \\ ORCID: https://orcid.org/0000-0001-7840-3246 \\ Faculdade Pernambucana de Saúde, Brasil \\ E-mail: andressa.carneiro08@hotmail.com \\ Marilia Mendes Vasconcelos \\ ORCID: https://orcid.org/0000-0003-4351-8363 \\ Centro Universitário Uninovafapi, Brasil \\ E-mail: mariliavascmendes@gmail.com \\ Renandro de Carvalho Reis \\ ORCID: https://orcid.org/0000-0002-3194-9767 \\ Centro Universitário Uninovafapi, Brasil \\ E-mail: renandro1981@hotmail.com
}

\begin{abstract}
Resumo
A telemedicina é a prestação de serviços remotos de saúde em situações na qual a distância é um fator crítico. Em meio ao cenário da COVID 19 a telemedicina sofreu muitos avanços, porém, ainda se observa entraves em sua aplicabilidade. Nesse contexto, o presente estudo teve por objetivo realizar uma revisão sistemática focando nas vantagens e entraves do uso da telemedicina no acompanhamento de pacientes com insuficiência cardíaca (IC). Para isso foi realizado um levantamento bibliográfico por meio da biblioteca eletrônica PubMed, tendo como critérios de inclusão artigos cujo tema central era o uso da telemedicina no acompanhamento de pacientes com IC publicados no período de 2017 a 2021. Foram identificados, no total, 95 artigos, dos quais apenas 13 tiveram contribuições relevantes para o presente estudo. Analisando-os foi identificado que o uso de serviços remotos em saúde proporciona aumento na adesão do paciente ao tratamento, reduzindo, assim, a taxa de hospitalização e morte, além de ter ótimo custo-benefício. No entanto, a impossibilidade de realização do exame físico e a falta de acesso a dispositivos eletrônicos e internet por parte da população são empecilhos que dificultam sua aplicabilidade. Portanto, apesar dos muitos benefícios proporcionados pela telemedicina para os pacientes com IC, é necessária uma logística bem estabelecida para que entraves sejam superados.
\end{abstract}

Palavras-chave: Telemedicina; Telemeonitoramento; Insuficiência cardíaca.

\begin{abstract}
Telemedicine is the remote delivery of health care services in situations that distance is a critical factor. Amidst of the COVID 19 scenario, telemedicine has undergone many advances, however, there are still obstacles in its applicability. In this context, the present study aims to conduct a systematic review focusing on the advantages and barriers of using telemedicine in monitoring patients with heart failure (HF). For this purpose, a bibliographic survey was carried out through the electronic library PubMed, having as inclusion criteria articles whose central theme was the use of telemedicine in monitoring patients with HF published between 2017 and 2021. In total, 95 articles were identified, of which only 13 had relevant contributions to the present study. Analyzing them, it was identified that the use of remote health services provides an increase in patient adherence to treatment, thus reducing the rate of hospitalization and death, in addition it has excellent cost-benefit. However, the impossibility of carrying out the physical examination and the population's lack of access to electronic devices and internet are obstacles that hinder its applicability. Therefore, despite the many benefits provided by telemedicine for patients with HF, well-established logistics are necessary for these barriers to be overcome.
\end{abstract}

Keywords: Telemedicine; Telemonitoring; Heart failure. 


\section{Resumen}

La telemedicina es la prestación de servicios de salud a distancia en situaciones en las que la distancia es un factor crítico. En medio del escenario COVID 19, la telemedicina ha experimentado muchos avances, sin embargo, aún existen obstáculos para su aplicabilidad. En este contexto, el presente estudio tiene como objetivo realizar una revisión sistemática centrada en las ventajas y barreras del uso de la telemedicina en el seguimiento de pacientes con insuficiencia cardíaca (IC). Para ello, se realizó una encuesta bibliográfica a través de la biblioteca electrónica PubMed, teniendo como criterio de inclusión artículos cuyo tema central fue el uso de la telemedicina en el seguimiento de pacientes con IC publicados en el período 2017 a 2021 . Un total de 95 artículos fueron identificados., de los cuales solo 13 tuvieron aportes relevantes para el presente estudio. Analizándolos, se identificó que el uso de servicios de salud a distancia proporciona un aumento en la adherencia del paciente al tratamiento, reduciendo así la tasa de hospitalización y muerte, además de tener una excelente relación costo-beneficio. Sin embargo, la imposibilidad de realizar el examen físico y la falta de acceso de la población a dispositivos electrónicos e internet son obstáculos que dificultan su aplicabilidad. Por tanto, a pesar de los múltiples beneficios que aporta la telemedicina a los pacientes con IC, es necesaria una logística bien establecida para superar los obstáculos.
\end{abstract}

Palabras clave: Telemedicina; Telemonitorización; Insuficiencia cardíaca.

\title{
1. Introdução
}

A telemedicina é a prestação de serviços remotos de saúde na promoção e prevenção de situações em que a distância seja um fator crítico (Organização Mundial da Saúde, 2019, Organização Pan Americana da Saúde 2019). Sua finalidade é fornecer um amplo benefício que vai desde os cuidados primários de cirurgias complexas, potencializa serviços médicos, amplia a atenção e a cobertura. Assim, os serviços disponibilizados em assistência remota, telerregulação, gestão administrativa e tecnologias de informações e comunicação (TIC) tem finalidade de melhorar o nível de atendimento em saúde promovendo a manutenção desses cuidados: diagnóstico, prevenção e tratamento (Lopes e Heimann, 2016).

A telemedicina avança em vários domínios, dentre eles, a Cardiologia tem sido a especialização que dispõe fácil acesso e sensibilidade na utilização de TICs de forma integrada na Atenção Básica à saúde. Dessa maneira, o uso da telemedicina em cardiologia pode representar o diferencial capaz de influenciar na melhoria da qualidade de atendimentos a doenças cardiovasculares, principalmente a hipertensão e a insuficiência cardíaca (Lopes et. al. 2019).

A insuficiência cardíaca (IC) é uma síndrome clínica complexa que apesar de seus conhecimentos crescerem cada vez mais, ainda há muitos desafios. Um desses desafios tem sido o tratamento para reduzir as taxas de internação e readmissão por agravamento da IC (Comitê coordenador da diretriz de insuficiência cardíaca, 2018; Koehler et al., 2018). Estima-se que a IC constitui cerca de $4 \%$ das internações gerais e atinge $31 \%$ das internações relacionadas a doenças cardiovasculares no Sistema Único de Saúde - SUS; o período de permanência, em média é de 5,8 dias e a mortalidade hospitalar varia entre 5,6 e 6\%. (Vasconcelos et al., 2020; Andrade et. al., 2013). Assim, a necessidade de diminuição da morbidade da IC auxiliado ao crescente desenvolvimento da telemedicina em cardiologia tem sido amplamente estudado a fim de verificar eficácia, vantagens e obstáculos. O objetivo desta revisão foi determinar as vantagens e entraves do telemonitoramento de pacientes com IC.

\section{Metodologia}

O presente estudo é uma revisão sistemática de caráter qualitativo, pois visa responder uma pergunta sobre um problema específico da área da saúde (Ercole, Melo e Alcoforado, 2014). O levantamento bibliográfico foi realizado em janeiro de 2021 por meio da biblioteca eletrônica PubMed. Essa base de dados foi a escolhida por ser direcionada à literatura médica. O Quadro 1 mostra a estratégia de busca utilizada nessa pesquisa. 
Quadro 1. Estratégia de busca avançada.

\begin{tabular}{|c|c|c|c|c|}
\hline $\begin{array}{c}\text { KEY WORD } \\
\text { DOCUMENT TITLE }\end{array}$ & $\begin{array}{c}\text { SEARCH } \\
\text { OPERATOR }\end{array}$ & $\begin{array}{c}\text { KEY WORD IN } \\
\text { DOCUMENT TITLE }\end{array}$ & $\begin{array}{c}\text { SEARCH } \\
\text { OPERATOR }\end{array}$ & $\begin{array}{c}\text { DATE } \\
\text { PUBLICATION }\end{array}$ \\
\hline \multirow{3}{*}{ Telemedicine } & \multirow{3}{*}{ AND } & $\begin{array}{c}\text { Telemedicine } \\
\text { Cardiology }\end{array}$ & \multirow{3}{*}{ AND } & \multirow{3}{*}{2017 to 2021} \\
\hline & & $\begin{array}{l}\text { Telemedicine } \\
\text { Heart Fealure }\end{array}$ & & \\
\hline & & $\begin{array}{l}\text { Benefits of } \\
\text { Telemonitoring } \\
\text { in Heart Fealure }\end{array}$ & & \\
\hline
\end{tabular}

Fonte: Autores.

Para realizar essa pesquisa, os artigos selecionados deveriam responder à pergunta de pesquisa "qual as vantagens e entraves da telemedicina na área da cardiologia?”. Os critérios de inclusão adotados foram os artigos cujo tema central fosse o uso da telemedicina no acompanhamento de pacientes com insuficiência cardíaca publicados no período de 2017 a 2021 . Os artigos encontrados na busca passaram etapas de análise dos resumos, seguido da leitura na íntegra (dos artigos selecionados após leitura dos resumos) e inclusão (após leitura do artigo na íntegra), aos quais foram distribuídos em tabela de Excel.

\section{Resultados e Discussão}

A Figura 1 mostra os passos seguidos nessa revisão sistemática. Os resultados estão disponibilizados na Tabela 01. Foram identificados 95 artigos, dos quais 58 eram duplicados ou com título irrelevante para a pesquisa. Por meio do abstract e do resumo foram selecionados 22, estes que foram lidos na íntegra, mas dos quais apenas 13 tiveram contribuições relevantes para o presente estudo. Os resultados foram classificados na tabela quanto à hospitalização, acessibilidade e custo.

Figura 1. Passos do presente estudo.

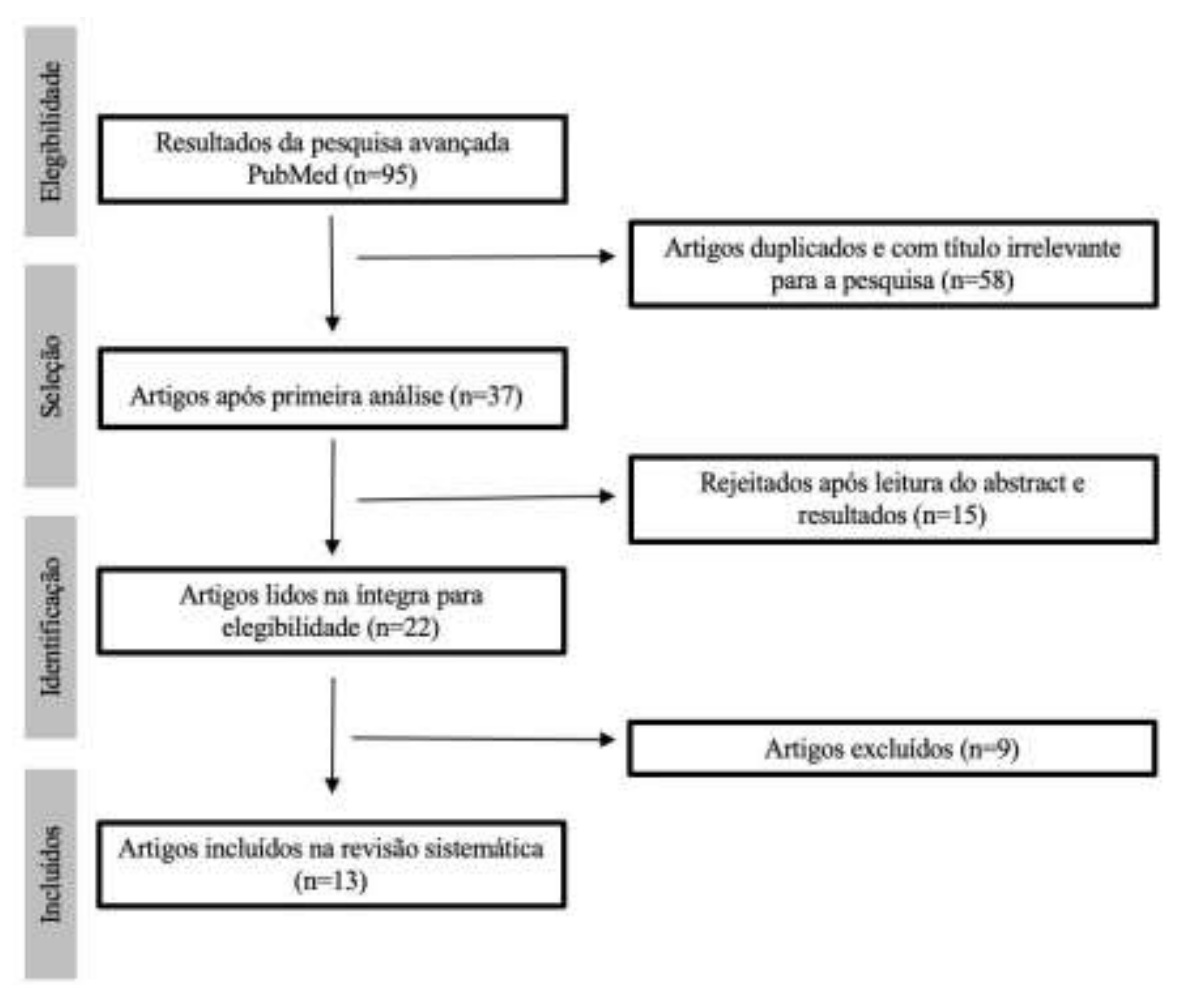

Fonte: Autores. Adaptado do fluxograma PRISMA statement (Moher, et al., 2010). 
A Tabela 1 mostra os achados das vantagens e dos entraves do uso da telemedicina no acompanhamento de pacientes com IC. Nela, tem-se a subdivisão em categorias conforme foram sendo identificadas, sendo elas: hospitalização, acessibilidade e custo. A identificação de onde cada resultado foi encontrada foi feita ao lado com os nomes dos respectivos autores.

Tabela 1. Vantagens e Entraves da telemedicina.

\begin{tabular}{|c|c|c|c|}
\hline Item & Vantagens $^{\mathrm{a}}$ & Entraves $^{\mathrm{b}}$ & Autores \\
\hline Hospitalização & $\begin{array}{l}\text { Redução das hospitalizações e mortes } \\
\text { relacionadas à insuficiência cardíaca }\end{array}$ & Insegurança médico-legal; & $\begin{array}{l}\text { KOEHLER et.al., } \\
2018^{\text {a; }} \text { ZHU, GU, } \\
\text { XU, } 2020^{\text {a. }} \text { CHAET } \\
\text { et. al. } 2017^{\text {b. }}\end{array}$ \\
\hline \multirow{3}{*}{ Acessibilidade } & $\begin{array}{l}\text { Aumento na adesão e participação dos } \\
\text { pacientes; }\end{array}$ & $\begin{array}{l}\text { Aplicação limitada por questões logísticas e } \\
\text { financeiras; }\end{array}$ & $\begin{array}{l}\text { GENSINI et. al. } 2017 \\
\text { a GENSINI et. al. } \\
2017^{\text {b. }}\end{array}$ \\
\hline & $\begin{array}{l}\text { Permite acesso à maior número de } \\
\text { pessoas; }\end{array}$ & $\begin{array}{l}\text { Questões de legislação referentes ao uso de } \\
\text { dados, à privacidade e à confidencialidade; }\end{array}$ & $\begin{array}{l}\text { GENSINI et. al. } 2017 \\
\text { a; EYCK et. al. } 2019 \\
\text { a; CHAET et. al. } \\
2017^{\text {b. }}\end{array}$ \\
\hline & $\begin{array}{l}\text { Permite o acompanhamento de pacientes } \\
\text { com insuficiência cardíaca mantendo-os } \\
\text { seguros do risco de infecções intra- } \\
\text { hospitalares (ex: COVID-19); }\end{array}$ & $\begin{array}{l}\text { Disponibilidade de internet e de dispositivos } \\
\text { eletrônicos. }\end{array}$ & $\begin{array}{l}\text { TERSALVI et. al. } \\
2020 \text { a; TERSALVI } \\
\text { et. al. } 2020^{\text {b }} \text {. }\end{array}$ \\
\hline Custo & $\begin{array}{l}\text { O maior engajamento dos pacientes ao } \\
\text { tratamento aponta uma redução } \\
\text { significativa dos custos médicos quando } \\
\text { comparado aos pacientes não engajados. }\end{array}$ & $\begin{array}{l}\text { A economia dos custos em programas de } \\
\text { telessaúde proporciona maior risco à saúde dos } \\
\text { pacientes (maior custo-efetivo em pacientes de } \\
\text { alto risco). }\end{array}$ & $\begin{array}{l}\text { EYCK et. al. } 2019 \text { a; } \\
\text { GENSINI et. al. } 2017 \\
\text { b. }\end{array}$ \\
\hline
\end{tabular}

Fonte: Autores.

As discussões pré pandêmicas sobre a telemedicina foram fundamentais para a implantação de uma assistência médica que permite um atendimento seguro e em condições críticas, como o que está ocorrendo no cenário atual da COVID 19. A telemedicina está associada, principalmente, à diminuição no número de hospitalizações (Brito, Leitão, 2020; Zhu, Gu, Xu, 2020).

Em estudo randomizado, a porcentagem de dias utilizados com hospitalizações por problemas cardíacos e mortes por todas as causas apresentou uma porcentagem de 4,88\% no grupo de tratamento remoto de pacientes e 6,64\% no grupo de tratamento usual. Além disso, o grupo de tratamento por teleconsulta perdeu, em média; 17,8 dias por ano quando em comparação ao grupo de pacientes de tratamento usual que perdeu em média 24,2 dias por ano em consultas. Por fim, a razão de risco para mortalidade de todas as causas foi de 0,70 (IC 95\% 0,50- 0,96; $\mathrm{p}=0,0280$ ) a favor do teleatendimento, porém, não houve uma diferença significativa em relação à mortalidade nos dois grupos estudados (Koehler, et. al. 2018).

No estudo randomizado realizado por Lin et. al. (2017), os resultados também são favoráveis para o uso da telemedicina no que tange todas as causas e a taxa de admissão hospitalar relacionada à IC, o tempo de internação e a 
mortalidade. Sendo que, a mortalidade geral por todas as causas (agrupados OR=0,80, IC 95\%0,71 a 0,91, p<0,001), taxa de admissão relacionada à IC (agrupados $\mathrm{OR}=0,63$, IC 95\% 0,53 a 0,76, p< 0,001) e HF- tempo de internação relacionado (diferença padronizada agrupada em médias $=-0,37$, IC de 95\% - 0,72 a - 0,02, p =0,041), foram bem menores quando comparado ao grupo controle. Reforçando, ainda mais, tais benefícios da telessaúde.

Nessa perspectiva, o enfrentamento de obstáculos como as burocracias, a situação financeira e a distância podem fazer com que o atendimento remoto se torne uma alternativa para pacientes com IC, aumentando a adesão desses, haja vista que as TICs oferecem a celeridade dos atendimentos, a reestruturação dos serviços de saúde por meio do favorecimento de competências e de especialidades que podem superar a distância física (um dos maiores desafios). No entanto, apresenta entraves por impossibilitar exames físicos e o maior custo em pacientes de alto risco, tendo em vista que, foi evidenciada a maior sensibilidade dos programas de telessaúde em pacientes de alto risco. Essa afirmativa é justificada pela possibilidade de médicos agendarem mais consultas virtuais futuras do que fariam presencialmente, aumentando custos imprevistos, apesar da pressuposta eficácia da telemedicina (Gensini, et. al.,2017; Maldonado, Marques, Cruz, 2016).

Além disso, pacientes engajados ao tratamento possuem custos médicos significativamente menores quando comparados aos pacientes não engajados. Portanto, tendo em vista que a telemedicina aumenta o comprometimento do paciente ao tratamento, é notório o custo-benefício do uso da telemedicina para pacientes com IC. O estudo realizado por Vestergaard reforça esse benefício ao ter como resultados uma redução de 35\% dos custos totais em saúde (Eyck, et. al., 2019; Vestergaard et. al.2020).

Ademais, consoante Chaet et al. (2017), a telemedicina também enfrenta entraves relacionados à insegurança quanto à privacidade e à confidencialidade do paciente, pois nos serviços de saúde virtual, há o envolvimento de terceiros para que os atendimentos sejam viabilizados, tais como serviços de telecomunicações e suas afiliadas. Desse modo, nem sempre informações podem ser seguras, a exemplo de sites que disponibilizam informações sobre saúde, causando, inclusive, o vazamento de dados dos usuários para terceiros, esse fator influencia na dificuldade de confiança dos pacientes na postura médico-legal.

Segundo Tersalvi et. al. (2020), os entraves estão presentes quanto à necessidade de posse, por parte dos pacientes, de instrumentos tecnológicos (celulares, computadores, tablets), limitação de acesso à internet para aqueles que residem em zona rural. Além disso, parte desses pacientes necessita da assistência de outra pessoa (familiar ou cuidador) no auxílio à conectividade com as teleconsultas. Porém, há vantagens no teleatendimento cardiológico por reduzir riscos de infecções, como a COVID-19. Possibilita, também, o tratamento de pacientes com IC avançada em aguardo de transplante com imunossupressão estável sem risco de rejeição de aloenxerto, pacientes com dispositivo de assistência ventricular esquerda (left ventricular assist device- LVAD) hemodinamicamente otimizado.

As intervenções de telemonitoramento que envolvam dispositivos automatizados e a característica móvel do telemonitoramento são as únicas formas de redução de risco de mortalidade por todas as causas e hospitalizações de IC, porém é necessário ter cuidado com essa informação e ser considerada como hipótese devido à natureza exploratória da própria pesquisa. A telemedicina vem avançando nas doenças crônicas juntamente com novos métodos, sendo eles: invasivo, não invasivo, com dispositivos móveis e imóveis. Todavia, devido à falta de comparações diretas entres essas opções, não é possível determinar qual é o método mais eficiente (Kitsiou, Paré, Jaana, 2015; Eurlings et. al., 2019).

Por fim, de acordo com Veenis et. al., 2021, o monitoramento remoto não invasivo é mais aconselhado em pacientes com IC crônica de risco menor ou pouco sintomático, porque é mais simples, de natureza não invasiva e com custos reduzidos. Ademais, como o número de pacientes com IC é elevado, o telemonitoramento não invasivo pode proporcionar grande impacto e a custos mais baixos. Já pacientes com IC de alto risco e mais sintomáticos, poderiam se beneficiar melhor de procedimentos de monitoramento remotos mais invasivos, no entanto, a custos mais altos. 


\section{Conclusão}

Percebe-se que as vantagens observadas dialogam com o contexto atual da COVID-19 por diminuir o fluxo de pessoas nos ambientes de saúde. Todavia, os entraves quanto ao exame físico e falta de logística na consulta com equipe multiprofissional são debates que precisam ser elevados e aprimorados. A implantação da telemedicina que, antes da pandemia, era apenas um debate, hoje é uma realidade que pode ter vindo para ficar.

Este trabalho reforça a ideia de que são necessárias estratégias de aperfeiçoamento no uso da telemedicina para o aprimoramento do atendimento cardiológico; portanto sugere-se mais pesquisas e protocolos bem estabelecidos que busquem minimizar os entraves apresentados no presente estudo.

\section{Referências}

Andrade, J. P. D., Mattos, L. A. P., Carvalho, A. C., Machado, C. A., \& Oliveira, G. M. M. D. (2013). Programa nacional de qualificação de médicos na prevenção e atenção integral às doenças cardiovasculares. Arquivos Brasileiros de Cardiologia, 100(3), $203-211$.

Brito, B. O., \& Leitão, L. P. C. (2020). Telemedicina no Brasil: Uma estratégia possível para o cuidado em saúde em tempo de pandemia?. Saúde em Redes, $6(2$ Suplem).

Chaet, D., Clearfield, R., Sabin, JE, \& Skimming, K. (2017). Prática ética em telessaúde e telemedicina. Journal of general internal medicine, 32 (10), 11361140 .

Ercole, F. F., Melo, L. S. D., \& Alcoforado, C. L. G. C. (2014). Revisão integrativa versus revisão sistemática. Revista Mineira de Enfermagem, $18(1)$, 9-12.

Eurlings, CGMJ, Boyne, JJ, De Boer, RA e Brunner-La Rocca, HP (2019). Telemedicina na insuficiência cardíaca - mais do que bom ter?. Netherlands Heart Journal, 27 (1), 5-15.

Eyck, L.T., MacLeod, S., Hawkins, K., Guimont, R., \& Hartley, S. (2019). The impact of a heart failure management program in a Medicare advantage population. Population health management, 22(2), 153-161.

Gensini, G. F., Alderighi, C., Rasoini, R., Mazzanti, M., \& Casolo, G. (2017). Value of telemonitoring and telemedicine in heart failure management. Cardiac failure review, 3(2), 116 .

Insuficiência, C. C. D. D., Colaboradores, C., \& Rohde, L. E. P. (2018). Diretriz Brasileira de Insuficiência Cardíaca Crônica e Aguda. Arq Bras Cardiol, 111(3), 436-539.

Kitsiou, S., Paré, G., \& Jaana, M. (2015). Efeitos das intervenções de telemonitoramento domiciliar em pacientes com insuficiência cardíaca crônica: uma visão geral das revisões sistemáticas. Journal of medical Internet research, 17 (3), e63.

Koehler, F., Koehler, K., Deckwart, O., Prescher, S., Wegscheider, K., Kirwan, B. A., ... \& Stangl, K. (2018). Efficacy of telemedical interventional management in patients with heart failure (TIM-HF2): a randomised, controlled, parallel-group, unmasked trial. The Lancet, $392(10152), 1047-1057$.

Lin, M. H., Yuan, W. L., Huang, T. C., Zhang, H. F., Mai, J. T., \& Wang, J. F. (2017). Clinical effectiveness of telemedicine for chronic heart failure: a systematic review and meta-analysis. Journal of Investigative Medicine, 65(5), 899-911.

Lopes, J. E., \& Heimann, C. (2016). Uso das tecnologias da informação e comunicação nas ações médicas a distância: um caminho promissor a ser investido na saúde pública. Journal of Health Informatics, $8(1)$.

Lopes, M. A. C. Q., Oliveira, G. M. M. D., Ribeiro, A. L. P., Pinto, F. J., Rey, H. C. V., Zimerman, L. I., ... \& Rezende, W. F. D. (2019). Guideline of the Brazilian Society of Cardiology on Telemedicine in Cardiology-2019. Arquivos brasileiros de cardiologia, 113(5), $1006-1056$.

Maldonado, J. M. S. D. V., Marques, A. B., \& Cruz, A. (2016). Telemedicina: desafios à sua difusão no Brasil. Cadernos de Saúde Pública, 32 , e00155615.

Moher, D., Liberati, A., Tetzlaff, J., \& Altman, D. G. (2010). Preferred reporting items for systematic reviews and meta-analyses: the PRISMA statement. Int J Surg, 8(5), 336-341.

Organização Mundial da saúde. (OMS). Digital Atlas Health. Internet. https://digitalhealthatlas.org/en//.

Organização Pan-Americana da Saúde. (OPS). Definição de indicadores para projetos de telemedicina como ferramenta para redução das iniquidades em saúde: documento de análise e resultados de uma comunidade de práticas. Washington D. C; 2016 [http://iris.paho.org/xmlui/handle/123456789/28563.

Tersalvi, G., Winterton, D., Cioffi, G. M., Ghidini, S., Roberto, M., Biasco, L., ... \& Vicenzi, M. (2020). Telemedicine in heart failure during COVID-19: a step into the future. Frontiers in cardiovascular medicine, 7.

Vasconcelos, H. G., da Silva Vaz, S. H., Prado, L. F. R., Rezende, L. C., Machado, A. P. B., Pereira, D. F. G., ... \& Andrade, V. F. (2020). Análise da mortalidade hospitalar por insuficiência cardíaca no estado de Minas Gerais, Brasil. Revista Eletrônica Acervo Científico, 12, e4568-e4568.

Veenis, JF, Radhoe, SP, Hooijmans, P., \& Brugts, JJ (2021). Monitoramento remoto em pacientes com insuficiência cardíaca crônica: o monitoramento remoto não invasivo é o caminho a percorrer ?. Sensores, 21 (3), 887. 
Research, Society and Development, v. 10, n. 9, e0410917708, 2021

(CC BY 4.0) | ISSN 2525-3409 | DOI: http://dx.doi.org/10.33448/rsd-v10i9.17708

Vestergaard, A. S., Hansen, L., Sørensen, S. S., Jensen, M. B., \& Ehlers, L. H. (2020). Is telehealthcare for heart failure patients cost-effective? An economic evaluation alongside the Danish TeleCare North heart failure trial. BMJ open, 10(1).

Zhu, Y., Gu, X., \& Xu, C. (2020). Effectiveness of telemedicine systems for adults with heart failure: a meta-analysis of randomized controlled trials. Heart failure reviews, 25(2), 231-243. 